\title{
THE SPHERICAL DRIFT CHAMBER FOR X-RAY IMAGING APPLICATIONS
}

\author{
G. CHARPAK, Z. HAJDUK*, A. JEAVONS, R. STUBBS \\ CERN, Geneva, Switzerland \\ and \\ R. $\mathrm{KHAN}^{\dagger}$ \\ LURE, Centre Universitaire, Orsay, 91405, France
}

Received 5 August 1974

Properties of proportional chambers with spherical drift spaces have been investigated.

An experiment of X-ray diffraction in crystals shows that an accuracy of $0.5 \mathrm{~mm}$, at $20^{\circ}$ inclination with respect to the axis

\section{Introduction}

In the early work on proportional wire chambers at CERN, in 1968 and $1969^{1}$ ), drift spaces were added to normal chambers. It was shown that free electrons could easily be transferred from the drift region into the wire chamber, and the advantage of the increase in effective chamber thickness and hence of efficiency for X-ray detection was noted.

Unfortunately, when the X-ray trajectory is not parallel to the drift field the increase in thickness leads

* On leave from Institute of Nuciear Physics, Cracow, Poland.

$\dagger$ On leave from Laboratoire de Physicochimie Structurale, Université Paris, Val de Marne, France.

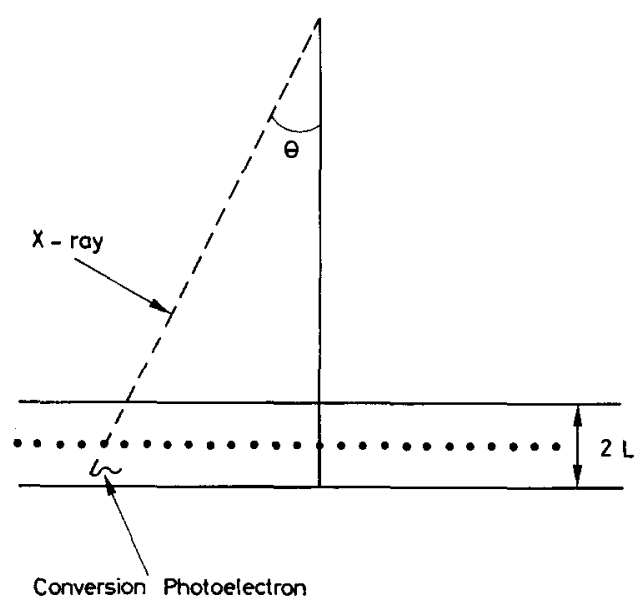

Fig. 1. Localization error for inclined directions of X-rays with flat chambers of thickness $2 L$; the position of the converted photon is distributed along a segment with projected length $2 L \operatorname{tg} \theta$. The range of the photoelectron released in the gas introduces another source of error, strongly dependent on its energy. of the chamber, can be obtained with $4 \mathrm{~cm}$ of drift length. Such chambers have many applications: X-ray diffraction patterns, pin-hole imaging, angular distributions of cascades of $\mathrm{X}$-rays in nuclear physics, etc.

to a serious degradation of resolution (fig. 1). In the case of pulsed X-ray sources, with very short time resolution, this can be overcome by measuring the delay in the detection of the pulse by the sense wires. This delay is proportional to the distance of the X-ray conversion point in the drift space to the sense wire plane. We present here a solution to this problem ${ }^{+}$for

+ We are indebted to Prof. R. Mössbauer, who raised the problem which led us to the solution of the spherical drift chamber, in the summer of 1973 . This solution was discussed at the Working Group Meeting on X-ray localization, organized by the European Molecular Biology Organization, in Heidelberg, 17th October 1973.

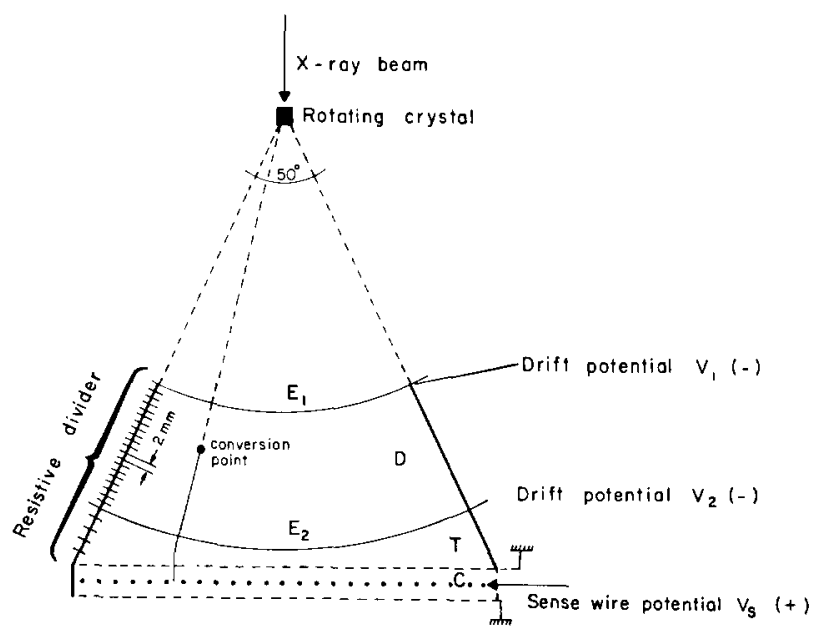

Fig. 2. Spherical drift chambers. The electrons converted in the drift space D are transferred to the multiwire chamber $C$, after transfer through the region $T$. The electric field is radial in $D$, with a proper choice of the potential distribution at the edge of the drift volume $\left(V_{2}-V_{1}\right) / V_{2}=0.66$. 
the case of continuous $X$-ray emission from a point source, e.g. X-ray diffraction from crystals, pin-hole imaging, etc. The drift space is spherical and the electric drift field is radial. Then all X-ray trajectories are parallel to the drift field, and full X-ray capture may be obtained without any loss of resolution.

Fig. 2 shows the construction of a chamber with an opening angle of $50^{\circ}$, built to test this idea. A drift space of $4 \mathrm{~cm}$ is enclosed by two spherical electrodes, the upper one made of $40 \mu \mathrm{m} \mathrm{Al}$, with a radius of $8.9 \mathrm{~cm}$, and the lower made with a mesh of $50 \mu \mathrm{m}$ stainless-steel wires spaced every $500 \mu \mathrm{m}$. In order to have spherical equipotentials over the largest possible drift volume, the conical epoxy edge of the volume is provided with conductive rings every $2 \mathrm{~mm}$ set at the potential corresponding to a radial field:

$$
V=\frac{r_{1} r_{2}}{\left(r_{2}-r_{1}\right)}\left|\left(\frac{1}{r_{1}}-\frac{1}{r}\right) V_{2}+\left(\frac{1}{r}-\frac{1}{r_{2}}\right) V_{1}\right| \text {. }
$$

This is done by a proper adjustment of a resistive divider.

At the typical working potential of $10 \mathrm{kV}$ applied at electrode $E_{1}$, the electric gradient is $1.8 \mathrm{kV} / \mathrm{cm}$ for the first centimeter after $E_{1}$ and $1.0 \mathrm{kV} / \mathrm{cm}$ for the last centimeter before the transfer grid $E_{2}$. The drifted electrons transfer to a $10 \times 10 \mathrm{~cm}^{2}$ flat multiwire chamber with $1 \mathrm{~mm}$ anode wire spacing, through the transfer space $\mathrm{T}$ (fig. 2). The two cathodes are made of $50 \mu \mathrm{m}$ wires, spread every $0.5 \mathrm{~mm}$, grouped into bands of $3 \mathrm{~mm}$ and perpendicular to each other. The read-out method, illustrated by fig. 3 and described elsewhere ${ }^{2}$ ), is based on the calculation of the centre

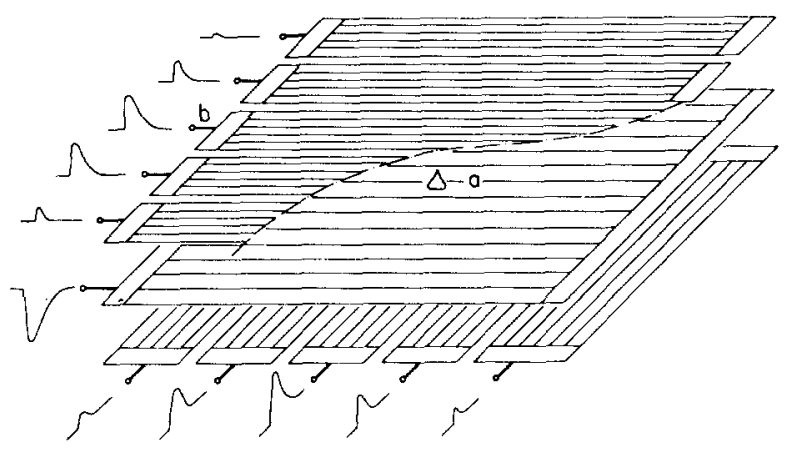

Fig. 3. Principle of the avalanche centre-of-gravity read-out method. The avalanches surrounding a wire $\mathbf{a}$ induce a positive pulse on the cathode strips $\mathbf{b}$. The pulse height of the induced pulse is measured and stored, and the centre of gravity of the pulse-height distribution is calculated and gives the position of the avalanche. The two cathode planes are equipped with strips parallel and orthogonal to the wires; coordinate $x$ parallel to the sense wires, coordinate $y$ orthogonal to the wires. of gravity of the avalanches, using the positive signals induced on each band of the cathode.

Fig. 4 shows a computer display of a spatial distribution of $6 \mathrm{keV} \mathrm{X}$-rays obtained by this method with the same chamber, without the drift space. It represents letters cut out of a plastic sheet.

The accuracy along the wire has been shown to be better than $300 \mu \mathrm{m}$ (fwhm), and an interpolating effect between the wires has been observed. It is not clear to what extent this may lead to an accuracy better than that of the wire spacing.

\section{Properties of the spherical chamber}

\subsection{EFFICIENCY AND ENERGY RESOLUTION}

The gas used in the chamber is a mixture of argon $(75 \%)$, isobutane $(23 \%)$, and methylal $(2 \%)$, flowing continuously. Some measurements were done with xenon replacing the argon. We tested the chamber mainly with $8 \mathrm{keV} \mathrm{Cu} \mathrm{K}$ line. The probability of absorption is about $63 \%$ in the drift space with argon and $100 \%$ with xenon. We observed, indeed, a considerable increase of efficiency with the drift voltage applied across the drift space.

The pulse-height distribution is peaked in a reasonably symmetric way showing that no major loss due to gas attachment occurs in the drift space. The resolution is better than $20 \%$ (fwhm) at $6 \mathrm{keV}$. The pulse height is reduced by the loss of free electrons, due to the limited transparencies of the transfer grids. This may be an advantage since it offers a possibility to

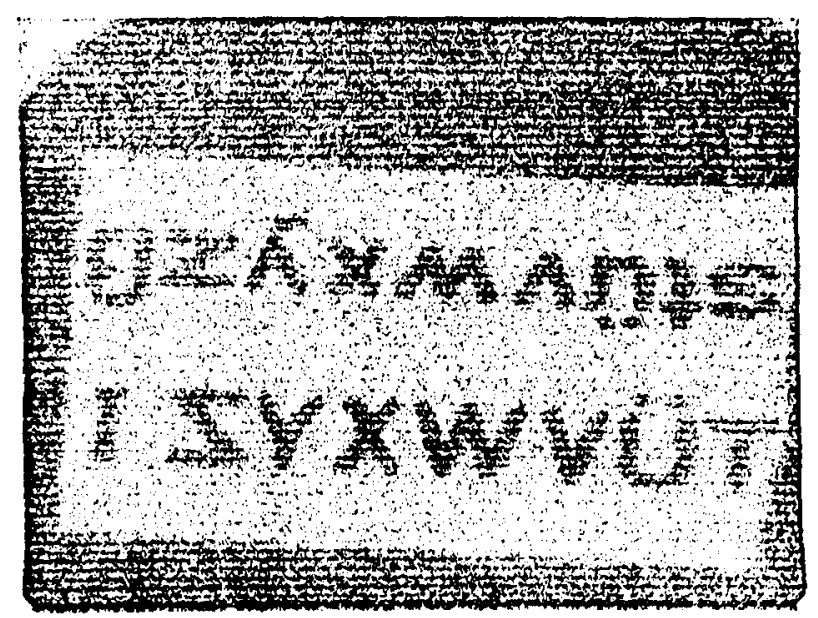

Fig. 4. An example of a spatial distribution of $X$-rays. Computer display obtained from a flat chamber, with $1 \mathrm{~mm}$ wire spacing, irradiated with $5.9 \mathrm{keV}$ X-rays. A thin plastic sheet with cut-out letters is placed against the chamber. Wire diameter $10 \mu \mathrm{m}$. Argon, isobutane, methylal filling. 
discriminate between the photons absorbed in the different parts of the chamber whenever the drift space does not totally absorb the X-rays.

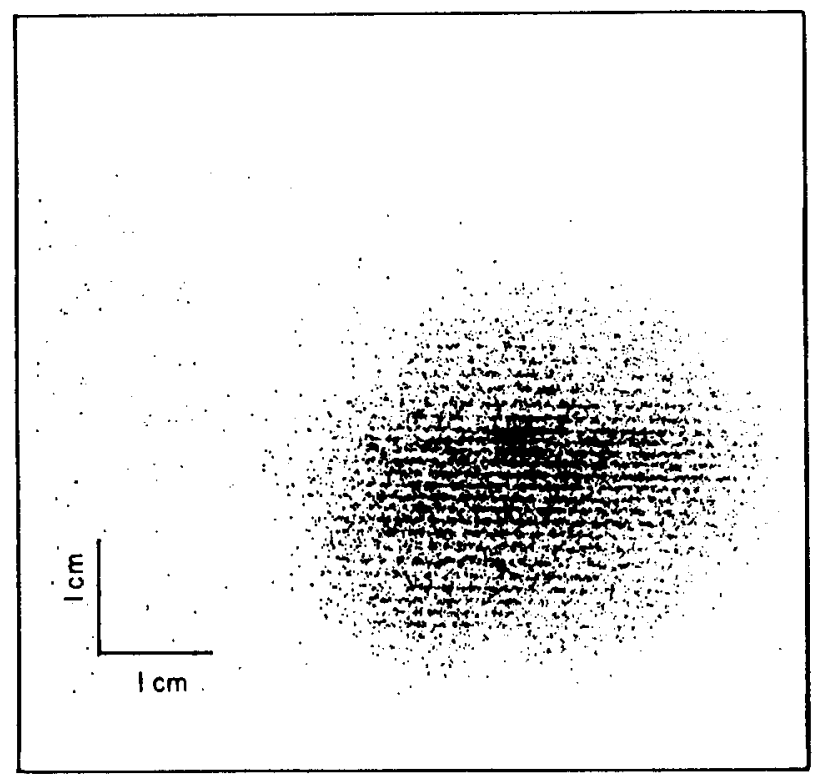

Fig. 5. Irradiation of a portion of the spherical chamber with an uncollimated source.

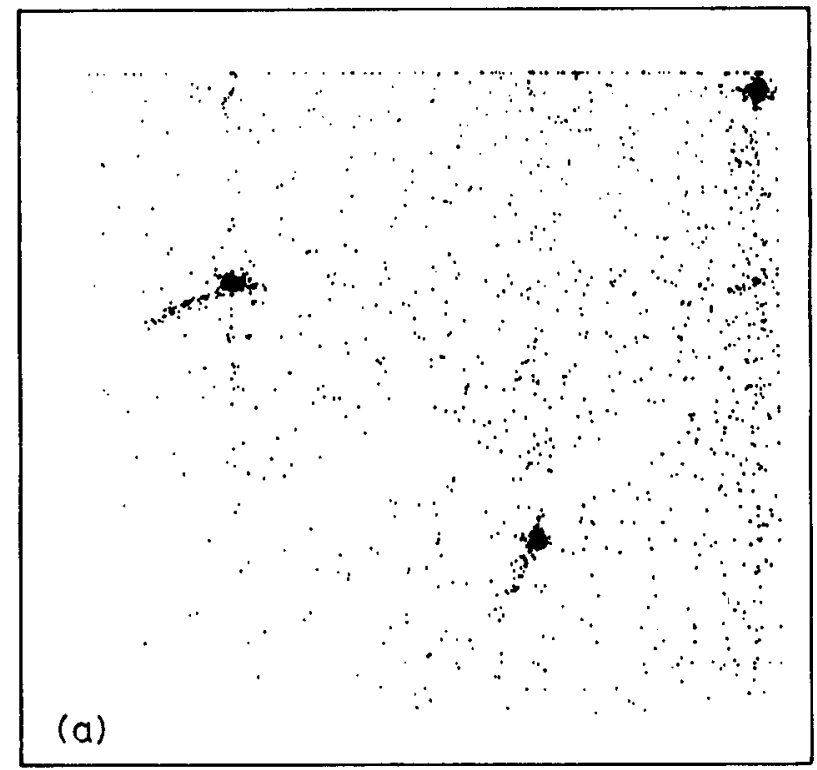

Fig. 6. Computer display of diffraction pattern obtained from an ambelline crystal $\mathrm{C}_{18} \mathrm{H}_{21} \mathrm{NO}_{5} 8 \mathrm{keV}$ X-ray beam. Total drift voltage $V_{1}=10 \mathrm{kV}$. $\mathrm{V}_{\mathrm{S}}=+3 \mathrm{kV}$.

(a) At top right, central beam; at bottom right and top left, two diffraction spots at different angular positions of the crystal.

\subsection{SPACE RESOlution}

Fig. 5 shows the wire structure when a portion of the chamber is irradiated with an uncollimated source.

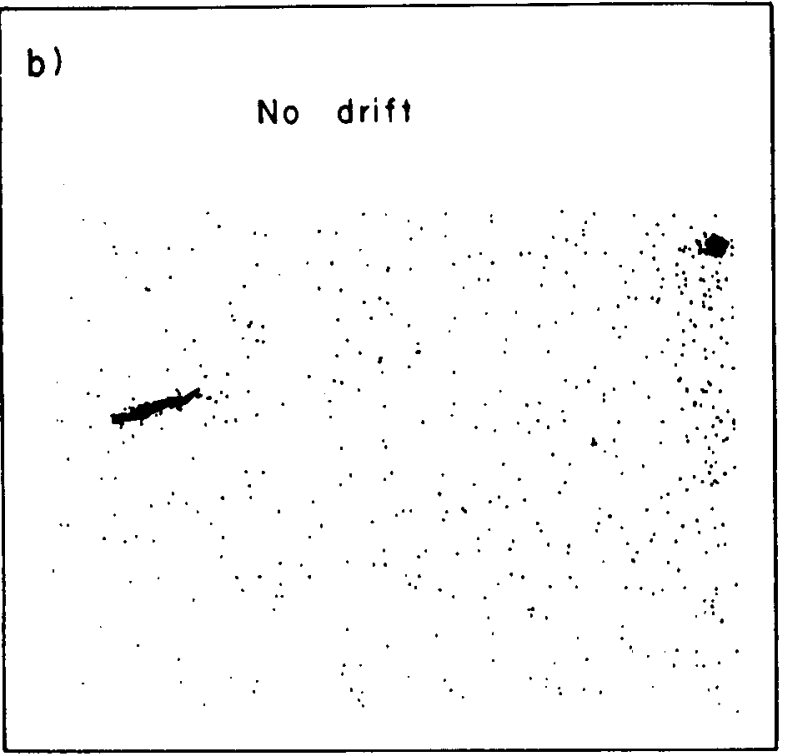

Fig. 6. (b) Photons traversing the drift space. The crystal is fixed at a position giving one diffraction spot. No potential is applied to the drift volume. Some electrons from the transfer region leak to the chamber.

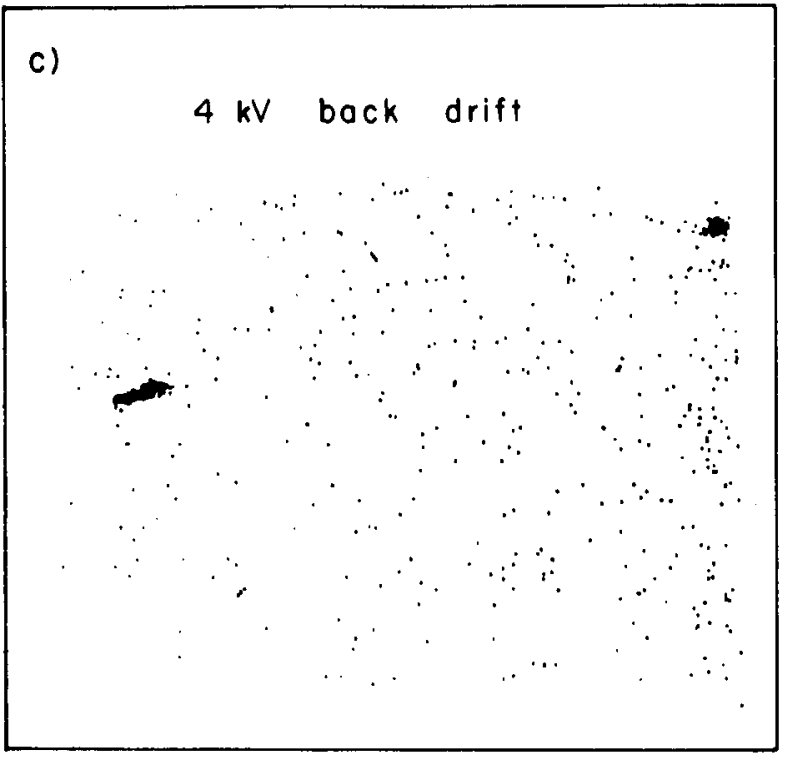

Fig. 6. (c) Same conditions as in (b) but a back potential is applied to the drift space: only photons absorbed in the chamber $\mathrm{C}$ are detected. We see the bad intrinsic resolution of a chamber with no spherical drift space. 
A collimated beam of $\mathrm{Cu} \mathrm{K}_{\alpha} \mathrm{X}$-rays was directed towards the centre of the chamber. A crystal of ambelline* of a size of a few tenths of a millimeter, rotates at the focus of the drift-space electrodes.

* $\mathrm{C}_{18} \mathrm{H}_{21} \mathrm{NO}_{5}$. Space group $\mathrm{P} 2_{1} 2_{1} 2_{1}: a=11.729 \AA, b=8.859 \AA$, $c=15.306 \AA$.
Fig. 6a shows a characteristic diffraction pattern, displayed by the computer. The spot at the upper right is produced by the direct beam and two reflections at different angles are shown.

The structure of the reflection spots is characteristic of a situation where a fraction of the X-rays is not absorbed in the drift space but in the transfer region $T$
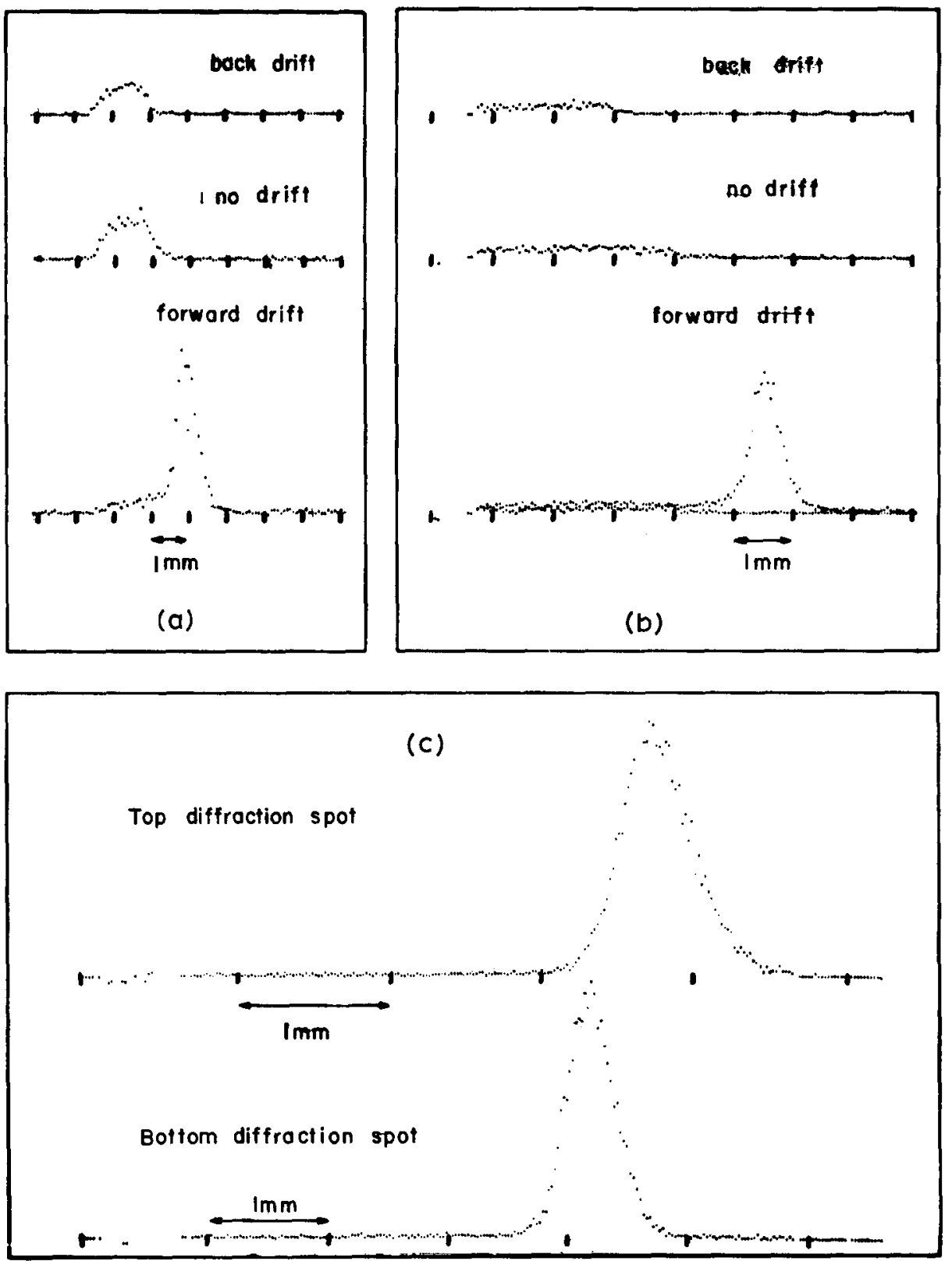

Fig. 7. Spatial distribution of the diffraction spots. The computer displays the intensity distribution along an axis parallel to the sense wires, separately for the two diffraction spots visible in fig. 6a. (a) Argon, isobutane, methylal filling. Bottom right spot, with a backward drift field $\left(V_{1}>0\right)$, no drift field $\left(V_{1}=V_{2}=0\right)$ and a forward drift field $\left(V_{1}=-10 \mathrm{kV}\right)$. (b) Same gas filling. Top left spot. The shape of the distributions corresponding to the absorption in the different volumes $\mathrm{D}, \mathrm{T}$, and $\mathrm{C}$ of fig. 2 are then clearly visible. (c) Xenon, isobutane, methylal filling. The two spots show the same structure; no photon traverses the drift region D. 
and in the chamber $C$. In figs. $6 \mathrm{~b}$ and $6 \mathrm{c}$ the crystal is set at a position where only one reflection appears. We see the result with the drift voltage switched off: the photons are detected directly in the chamber or in the transfer region. Fig. $6 \mathrm{c}$ shows the display with a back-drift voltage applied to the transfer drift region, and we detect only the photons absorbed in the chamber $C$. We see clearly the gain in resolution obtained from the spherical structure.

In order to measure only the photons absorbed in the drift region, two alternatives are possible: either the drift space is made absorptive enough by a correct choice of thickness and gas, or the pulse height is used to discriminate between photons absorbed in different regions (see section 2.1).

From the computer we can obtain a display of the intensity distribution along any direction. In fig. 7 are seen the distributions of photon intensities along the axis parallel to the wires, separately for each of the spots seen in fig. 6a. The fwhm of $550 \mu \mathrm{m}$ obtained with argon fillings reflects the range of the photoelectrons in argon and the size of the crystal. We have repeated this measurement with xenon replacing argon (fig. $7 \mathrm{c}$ ). We see that the tails due to the photons absorbed in the transfer region have disappeared and that the resolution, $470 \mu \mathrm{m}$, is slightly improved, owing to the reduction of the photoelectron range.

\subsection{SigNAL-TO-NOISE RATIO}

It should be emphasized that the signal-to-noise ratio for a rotating crystal is much more favourable

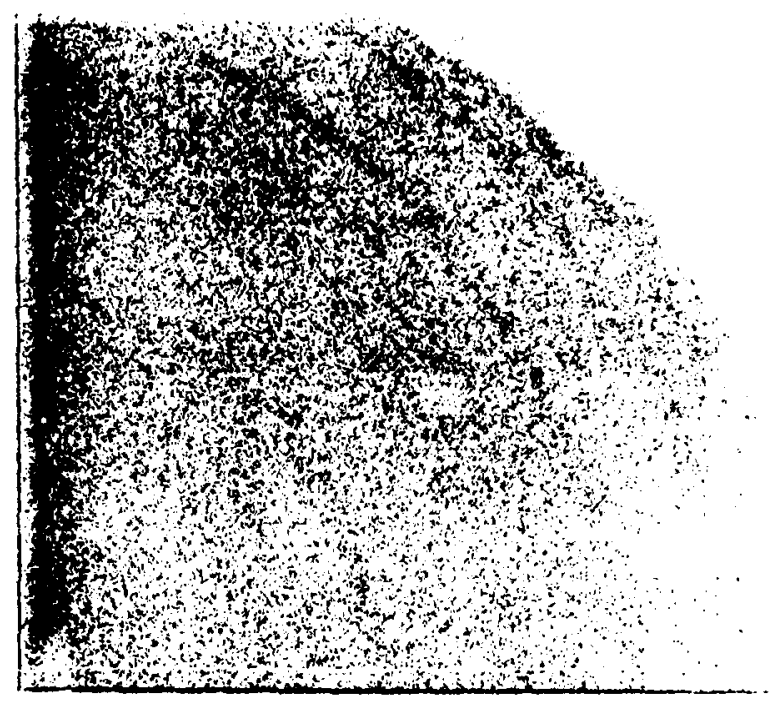

Fig. 8. Diffraction pattern of a powder. The considerable noise is due to the glass support for the powder. with such a method than with a detection method, such as film, where the background is integrated over $360^{\circ}$. The computer can keep a record of the time of detection of the photon, and only those background counts that have been detected while the crystal is at a position where it detects a reflection are to be taken into account. The noise in a proportional chamber is known to be about 1 count per meter of wire, per second. This includes the cosmic-ray background and the ambient radioactivity.

The X-ray offers two additional ways of reducing this background. One is the energy discrimination, the other one is the use of the pattern of sense wires associated with every event. For X-rays the number of sense wires hit will be very limited, while it may be considerable with high-energy electrons coming from the background. This is more so with a large drift space.

A diffraction pattern obtained with a powder is shown in fig. 8. The considerable noise is produced by the glass support for the powder, as was checked with a film. Some circular lines are clearly visible.

\section{Conclusion}

Our measurements show that spherical drift chambers present great advantages for the imaging of X-rays: diffraction by point crystals, imaging with pin-hole screens, etc. They permit a high increase in efficiency with no apparent loss of resolution. Low-cost gases such as argon can be used, with a large thickness compensating for the relatively low X-ray absorption coefficients. It is known that drift lengths of as much as $50 \mathrm{~cm}$ have been used in particle physics ${ }^{3}$ ) with accuracies showing that the diffusion still permits localization in the millimeter range.

The spherical drift spaces can be associated with multiwire chambers with any read-out methods.

Two read-out methods are actively developed for X-ray imaging with proportional chambers: the delayline method of the Perez-Mendez group, the resistive wire read-out used by Borkowski and $\mathrm{Kopp}^{4}$ ).

However, one may wonder whether the full exploitation of the data from a spherical drift chamber, particularly in those cases where the crucial problem is the signal-to-noise ratio, is not best carried out with digital methods giving also the number of sense wires involved in eacin event.

We are indebted to $\mathrm{Mr} \mathrm{R}$. Benoît who has, with great enthusiasm and cleverness, solved the technical problems encountered in the construction of the prototype, with the help of $\mathrm{Mr} \mathrm{N}$. Gregoric. 


\section{References}

1) G. Charpak, D. Rahm and H. Steiner, Nucl. Instr. and Meth. 80 (1970) 13.

2) G. Charpak, A. Jeavons, F. Sauli and R. Stubbs, CERN 73-11 (1973).
3) R. Chaminade, S. C. Duchazeaubeneix, C. Lapostalles and S. Saudinos, Nucl. Instr. and Meth. 111 (1973) 77.

4) All references concerning the latest stage of these two methods can be found in the IEEE Trans. Nucl. Sci. NS-21, no. 1 (1974). 